\title{
Sea ice mapping method for SeaWinds
}

David G. Long

david_long@byu.edu

Hyrum S. Anderson

Follow this and additional works at: https://scholarsarchive.byu.edu/facpub

Part of the Electrical and Computer Engineering Commons

\section{Original Publication Citation}

Anderson, H. S., and D. G. Long. "Sea Ice Mapping Method for SeaWinds." Geoscience and Remote Sensing, IEEE Transactions on 43.3 (25): 647-57

\section{BYU ScholarsArchive Citation}

Long, David G. and Anderson, Hyrum S., "Sea ice mapping method for SeaWinds" (2005). Faculty Publications. 391.

https://scholarsarchive.byu.edu/facpub/391 


\title{
Sea Ice Mapping Method for SeaWinds
}

\author{
Hyrum S. Anderson, Student Member, IEEE, and David G. Long, Senior Member, IEEE
}

\begin{abstract}
A sea ice mapping algorithm for SeaWinds is developed that incorporates statistical and spatial $a$ priori information in a modified maximum a posteriori (MAP) framework. Spatial a priori data are incorporated in the loss terms of a Bayes risk formulation. Conditional distributions and priors for sea ice and ocean statistics are represented as empirical histograms that are forced to conform to a set of expected histograms via principal component filtering. Tuning parameters for the algorithm allow adjustments in the algorithm's performance. Results of the algorithm exhibit high correlation with the Remund-Long sea ice mapping algorithm for SeaWinds and the Special Sensor Microwave/Imager National Aeronautics and Space Administration Team 30\% ice edge, and are verified with RADARSAT-1 ScanSAR imagery. The resulting sea ice maps exhibit high edge detail, preserve polynyas and ice bodies disjoint from the primary ice sheet, and thus are suitable for use with wind retrieval and sea ice studies. Principles employed in the algorithm may be of interest in other classification studies.
\end{abstract}

Index Terms-Bayes method, maximum a posteriori (MAP), principal components, QuikSCAT, scatterometer, sea ice extent, SeaWinds.

\section{INTRODUCTION}

$\mathbf{T}$ HE polar sea ice regime is an extremely dynamic environment that plays a crucial role in many geophysical processes. Sea ice covers from 15 to 23 million $\mathrm{km}^{2}$ of the earth's surface, fluctuating in response to seasonal climate conditions. Sea ice in the Northern Hemisphere doubles in area from fall to spring, extending into the mid-latitudes. The total sea ice area in the Southern Hemisphere is nearly four times greater in the austral spring than in the austral fall. Polar sea ice significantly increases the surface albedo in these areas and is a key factor in the earth's global radiation budget. In addition, polar sea ice is an excellent thermal insulator and physical barrier to the exchange of gases between the ocean and atmosphere. Atmospheric heat exchange over sea ice is up to two orders of magnitude less than exchange over open ocean.

Microwave sensors provide an excellent solution for remotely monitoring polar sea ice. They offer several advantages over visible or infrared alternatives. Microwaves are much less sensitive to atmospheric attenuation and distortion. This feature is particularly attractive in the polar regions where frequent and extensive cloud cover forms an opaque optical barrier in the atmosphere. In addition, microwave sensors do not require sunlight for illumination so that even during the sunless polar winters, data may be collected. The application of active microwave

Manuscript received January 23, 2004; November 23, 2004. This work was supported by the National Aeronautics and Space Administration.

H. S. Anderson was with the Center for Remote Sensing, Brigham Young University, Provo, UT 84602 USA. He is now with the Lincoln Laboratory, Massachusetts Institute of Technology, Lexington, MA 02420 USA.

D. G. Long is with the Center for Remote Sensing, Brigham Young University, Provo, UT 84602 USA (e-mail: long@ee.byu.edu).

Digital Object Identifier 10.1109/TGRS.2004.842017 sensors in particular to monitoring the cryosphere has provided over 25 years of polar sea ice data, with each new instrument providing increasingly higher temporal and spatial resolution. Several studies have verified the utility of microwave scatterometer data in polar sea ice detection and classification [1]-[4].

The Remund-Long (RL) sea ice extent algorithm for SeaWinds [3] — an adaptation of an earlier version for the National Aeronautics and Space Administration (NASA) Scatterometer (NSCAT) [4]-incorporates an iterative maximum likelihood discrimination scheme to statistically segment sea ice and ocean. Statistics for multivariate microwave signatures of sea ice are estimated from a nearest neighbor classification. Maximum-likelihood (ML) discrimination is iteratively applied to segment ice and ocean populations statistically. This results in an initial sea ice estimate that generally contains residual noise caused by wind-roughend ocean surfaces or other microwave signature anomalies. The RL algorithm filters these artifacts through a sequence of binary processing operations and sea ice growth/retreat constraints using sea ice extent information from a previous day.

The RL sea ice extent algorithm for SeaWinds has been successfully applied to enhanced resolution scatterometer data for both science data and near real-time (NRT) products. It is currently in operational use with NRT data by the National Oceanic and Atmospheric Administration for polar sea ice mapping and by the SeaWinds project for wind retrieval. The algorithm applied to the several years of enhanced-resolution SeaWinds data now available shows that the binary processing stage of the RL algorithm corrects the majority of the residual errors in the initial classification. However, the binary processing phase of the algorithm reduces the detail of the ice map via filtering and disallows sections of sea ice that are disjoint from the main ice sheet.

This paper presents a modified Bayes detection approach to segment polar sea ice and ocean using imagery derived from the SeaWinds instrument. The proposed algorithm has its roots in the RL sea ice extent algorithm for SeaWinds, using a multivariate statistical segmentation approach with the same discrimination parameters as the RL algorithm. However, the algorithm differs from the RL approach in several areas. First, statistical a priori estimates of sea ice and ocean are incorporated. Second, spatial a priori information is applied in the initial classification phase, as opposed to a postprocessing phase (growth/retreat constraints of the RL algorithm). Further, the RL algorithm implicitly assumes that the discrimination parameters are jointly Gaussian, whereas the proposed algorithm incorporates a data-driven empirical model that is constrained by a set of previously observed distributions. While specifically applied to SeaWinds data, the histogram modeling technique may be interesting for other sensors or classification studies.

The SeaWinds instrument and data products are discussed in Section II. The modified Bayes method for segmenting sea ice 
and ocean is developed in Section III with results in Section IV. A brief conclusion follows.

\section{BACKGROUND}

This section provides an introduction to the SeaWinds instrument. Data received from the SeaWinds instrument are gridded using the scatterometer image reconstruction (SIR) algorithm, which is also described here.

\section{A. SeaWinds Instrument}

The first SeaWinds instrument was launched aboard QuikSCAT (QSCAT) in June 1999 to fill the Ku-band data gap left by the failure of the NASA Scatterometer (NSCAT). In December 2002, a second SeaWinds instrument aboard the Advanced Earth Observing Satellite II (ADEOS-II) was launched, though ADEOS-II failed after only ten months of operation.

The SeaWinds instrument is a $13.4-\mathrm{GHz}$ dual-polarization scanning pencil beam scatterometer with two spot beams which are conically scanned [5] so that the $25 \times 37 \mathrm{~km}$ footprint of each antenna's field-of-view sweeps out wide, overlapping swaths. The outer beam ( $54^{\circ}$ incidence) measures the vertically polarized (v-pol) normalized radar cross-section $\left(\sigma^{\circ}\right)$, while the inner beam ( $46^{\circ}$ incidence) measures horizonatally polarized (h-pol) $\sigma^{\circ}$. As the platform traverses, the outer beam forms an $1800-\mathrm{km}$ swath and the inner beam forms a $1400-\mathrm{km}$ swath, each with no nadir gap. Consequently, the SeaWinds instrument covers nearly $90 \%$ of the earth's surface each day and all of the polar regions, excepting a gap directly over the poles due to the orbit geometry. This extent of spatial coverage and temporal resolution is ideal for observing polar sea ice, where floes can move as many as $25 \mathrm{~km}$ in a single day.

The SeaWinds instrument operates in two spatial resolution modes [6]. The intrinsic elliptical measurement cells, called "eggs" have a nominal resolution of $25 \mathrm{~km}$ in azimuth by $37 \mathrm{~km}$ in the range direction. The ice mapping method may be adapted for the higher resolution mode. In this mode, the "egg" cells are resolved into "slices" through range and Doppler processing. Egg data are used in this work, while the higher resolution slice data are left for future research.

\section{B. SIR Image Generation}

In this paper, images are generated with the aid of the scatterometer image reconstruction (SIR) algorithm [7], [8], though other algorithms could be employed. SIR is an iterative technique that uses samples from multiple satellite swaths to enhance the intrinsic resolution of the sensor, in effect, trading temporal resolution for spatial resolution. For polar regions, swaths over a 24-h imaging interval are used for image reconstruction. SeaWinds egg imagery gridded using the SIR algorithm is enhanced to $4.45 \mathrm{~km}$ pixel resolution, with an effective resolution of $8-10 \mathrm{~km} .{ }^{1}$

The SIR algorithm produces several image products derived from the SeaWinds instrument. Of interest in this study are $A_{\mathrm{v}}$, $A_{\mathrm{h}}, V_{\mathrm{v}}$, and $V_{\mathrm{h}} . A_{\mathrm{v}}$ and $A_{\mathrm{h}}$ are the enhanced resolution $\sigma^{\circ}$ images for v-pol and h-pol, respectively. Each pixel represents an

${ }^{1}$ SIR science data products for both egg and slice measurements are available at the Scatterometer Climate Pathfinder website http://www.scp.byu.edu. "average" $\sigma^{\circ}$ measurement over the 24-h interval from multiple swaths and from multiple looks in the same swath (i.e., azimuthal modulation contributes to the average). $V_{\mathrm{v}}$ and $V_{\mathrm{h}}$ are v-pol and h-pol standard deviation estimates of these same measurements.

The $A$ imagery exhibits good statistical contrast between sea ice and ocean. Rough surface scattering and volume scattering in sea ice contribute to an overall higher backscatter over sea ice than over the ocean, for which surface scattering dominates. The $V$ imagery also exhibits contrast because $\sigma^{\circ}$ measurements of the ocean show high temporal variability between successive satellite swaths and are affected by azimuth modulation from ocean waves. These effects are relatively small over sea ice, which exhibits lower diurnal variations and greater isotropy.

Derived from the image products is the quasi-copolarization ratio (PR), which is defined to be the ratio (difference in log-space) of $A_{\mathrm{V}}$ and $A_{\mathrm{h}}$, which are collected at different incidence angles. The quasi PR couples two microwave dependencies: incidence angle dependence (h-pol and v-pol differ by $8^{\circ}$ ), and polarization dependence. Measurements of $\sigma^{\circ}$ from sea ice backscatter show smaller incidence angle dependence than ocean due to rough surface and volume scattering. In addition, $\sigma^{\circ}$ measurements of ocean exhibit a v-pol bias, unlike $\sigma^{\circ}$ measurements of sea ice. These microwave dependencies contribute to make PR a good discriminant between sea ice and ocean. PR is high for ocean and low for sea ice and has been used in other sea ice extent algorithms (e.g., [9]).

\section{SeA ICE Mapping Algorithm}

Four image products-PR (derived), $A_{\mathrm{h}}, V_{\mathrm{v}}$, and $V_{\mathrm{h}}$-are used in concert as discrimination parameters to detect sea ice. Since it is helpful to incorporate a priori information in an estimate of polar sea ice extent, the algorithm is developed within the framework of Bayes detection, where a decision rule $\phi(\mathbf{z})$ selects sea ice or ocean based on an observation $\mathbf{z}$, and depending on the Bayes risk $R(\cdot)$ associated with that decision

$$
\phi(\mathbf{z})= \begin{cases}\text { sea ice, } & \text { if } R(\text { sea ice } \mid \mathbf{z})<R(\text { ocean } \mid \mathbf{z}) \\ \text { ocean, } & \text { otherwise. }\end{cases}
$$

The algorithm is designed for pixel-based classification, in which observations $\mathbf{z}=\left[\mathrm{PR} A_{\mathrm{h}} V_{\mathrm{v}} V_{\mathrm{h}}\right]$ for each pixel at position $(x, y)$ and time $t$ (in days) are used to determine the Bayes risk. Bayes risk is related to the probability $P(\cdot)$ that a hypothesis $H_{i}^{x, y, t}$ (the pixel belongs to class $i$ ) is correct given the observation $\mathbf{z}$. The decision rule selects the hypothesis $\left(H_{1}^{x, y, t}\right.$ for sea ice and $H_{0}^{x, y, t}$ for ocean) that results in a minimum expected value of Bayes risk

$$
R\left(H_{i}^{x, y, t} \mid \mathbf{z}\right)=P\left(H_{i}^{x, y, t} \mid \mathbf{z}\right) L_{i i}^{x, y, t}+P\left(H_{j}^{x, y, t} \mid \mathbf{z}\right) L_{i j}^{x, y, t}
$$

where $L_{i j}^{x, y, t}$ is an arbitrarily assigned loss for selecting $H_{i}^{x, y, t}$, given that $H_{j}^{x, y, t}$ (the pixel belongs to class $j$ ) is correct. For MAP criterion, $L_{i j}^{x, y, t}$ is zero for $i=j$ and unity for $i \neq j$. We will relax the latter constraint and require only $0 \leq L_{i j}^{x, y, t} \leq 1$ for $i \neq j$. In practice, it is difficult and sometimes impossible to calculate the probability in (2) for every pixel. In image processing, a common practice is to treat the pixel statistics as spatially uniform, i.e., a pixel's statistical behavior can be approximated by the statistics of all pixels in the image. With this 


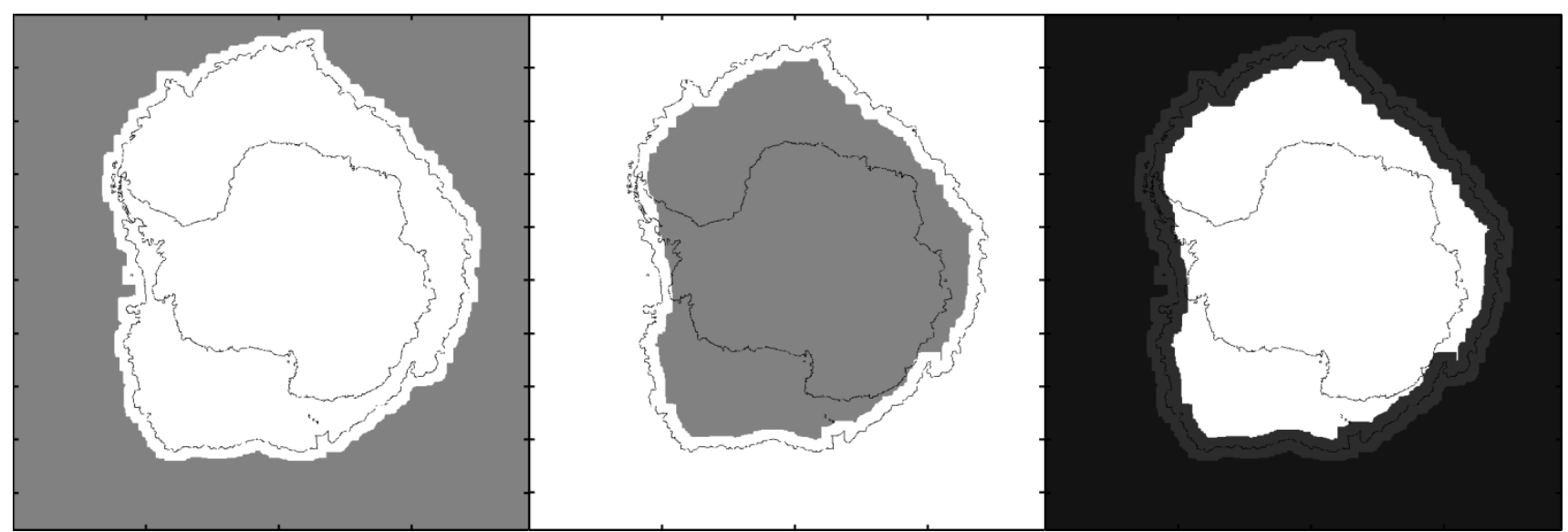

Fig. 1. Antarctic (left) ocean loss map $L_{01}$, (middle) ice loss $L_{10}$ map, (right) default decision rule showing the region of ignorance around the ice edge. In each case, dark shades represent low values, while light shades represent high values. Outlines are added for clarity. The algorithm may be applied to both the Arctic and Antarctic regions.

simplification, but retaining the general form for the loss, (2) reduces to

$$
R\left(H_{i}^{x, y, t} \mid \mathbf{z}\right)=P\left(H_{j}^{t} \mid \mathbf{z}\right) L_{i j}^{x, y, t} .
$$

In this form, inaccuracy introduced by neglecting spatial dependence in the $P\left(H_{j}^{t} \mid \mathbf{z}\right)$ may be accounted for by careful selection of $L_{i j}^{x, y, t}$.

Equation (3), written in terms of the posteriori probability, may be expressed in terms of the a priori probability using Bayes rule. To facilitate a binary decision (sea ice or ocean) using a likelihood ratio test, Bayes risk is expressed in terms of a conditional distribution [10]. This results in an effective decision rule $\phi(\mathbf{z})$ for an observation $\mathbf{z}$

$$
\phi(\mathbf{z})=\left\{\begin{array}{cc}
1, & \text { if } f\left(\mathbf{z} \mid H_{1}^{t}\right) P\left(H_{1}^{t}\right) L_{01}^{x, y, t} \\
& >f\left(\mathbf{z} \mid H_{0}^{t}\right) P\left(H_{0}^{t}\right) L_{10}^{x, y, t} \\
0, & \text { otherwise. }
\end{array}\right.
$$

In this formulation, $\phi(\mathbf{z})$ selects 1 if sea ice is more likely, and 0 otherwise. The decision threshold is adjusted by the priors and the costs associated with the decision. Rather than selecting a functional form for $f\left(\mathbf{z} \mid H_{i}^{t}\right)$, the conditional distributions are approximated using histograms, $h\left(\mathbf{z} \mid H_{i}^{t}\right)$, to allow for arbitrary distributions for sea ice and ocean. Equation (4) becomes

$$
\phi(\mathbf{z})= \begin{cases}1, & \text { if } h\left(\mathbf{z} \mid H_{1}^{t}\right) L_{01}^{x, y, t}>h\left(\mathbf{z} \mid H_{0}^{t}\right) L_{10}^{x, y, t} \\ 0, & \text { otherwise. }\end{cases}
$$

Note that the prior probabilities are reflected in the relative histogram integrals.

The Bayes detection formulation in (5) allows for a simple pixel-based test of ensemble sea ice and ocean histograms [fourdimensional (4-D)], where the loss terms essentially shift the threshold of the decision. The choice to assume spatial uniformity for the probability, but not for the loss terms in (2), is somewhat cavalier but will be justified in Sections III-A to III-D.

\section{A. Loss Maps}

The value assigned to $L_{i j}^{x, y, t}$ can be arbitrarily chosen. It is intended to quantify the consequence of a detection, either a correct or a incorrect one. Here, it is used to reflect the a priori belief that the hypothesis $H_{i}^{x, y, t}$ is correct. The relative values of the loss terms in the decision rule of (5) shift the decision threshold by scaling the histograms. Using $L_{i j}^{x, y, t}$ as a measure of prior belief, we may relate it to the probability that the observed pixel on day $t$ belongs to class $i$, given that it belonged to class $j$ the previous $(t-1)$ day; in effect

$$
L_{i j}^{x, y, t} \sim P\left(H_{i}^{x, y, t} \mid H_{j}^{x, y, t-1}\right)
$$

where a low value of probability is associated to any change in the map and a high value of probability to unchanged pixels. Expressed this way, the loss term functions to constrain the prior probabilities $P\left(H_{j}^{x, y, t}\right)$ that are considered to be dependent on the classification of a pixel the day before.

We implement $L_{i j}^{x, y, t}$ as a lookup table that retrieves a loss value for pixel location $(x, y)$ (for convenience, the values are restricted to the range $[0,1])$. For sea ice mapping, we set $L_{i j}^{x, y, t}$ based on sea ice extent maps from the previous day. Since the ice edge may move several kilometers in a single day and the sea ice extent map from the previous day may over- or underrepresent the true sea ice edge, we allow for sea ice growth and imperfect sea ice maps. Thus, the ocean loss factor $L_{01}^{x, y, t}$ is generated using a dilated version of the previous day's sea ice extent map. Likewise, the sea ice loss factor $L_{10}^{x, y, t}$ is generated using a dilated version of its complement (an ocean extent map). The sea ice loss lookup table is filled with high values (near unity) where yesterday's dilated ocean mask predicts ocean. It contains low values (near zero) where yesterday's dilated ocean mask predicts sea ice. Conversely, the ocean loss lookup table is filled with high values where yesterday's dilated ice mask predicts sea ice, and low values where yesterday's dilated ice mask predicts ocean.

The ratio of the loss maps $L_{01}^{x, y, t} / L_{10}^{x, y, t}$ adjusts the threshold for the MAP-like decision rule in (5). This ratio determines the classification result if the bin heights of $h\left(\mathbf{z} \mid H_{1}^{t}\right)$ and $h\left(\mathbf{z} \mid H_{0}^{t}\right)$ for an observed $\mathbf{z}$ are equal. The dilation procedures used in forming the loss maps create a region where equal loss is assigned to both sea ice and ocean. In this region, where $L_{01}^{x, y, t}=$ $L_{10}^{x, y, t}$ (i.e., spatial a priori information is inconclusive), the unweighted ratio of the sea ice and ocean histograms determines the classification. Sample loss maps and the corresponding default decision rule are shown in Fig. 1. 

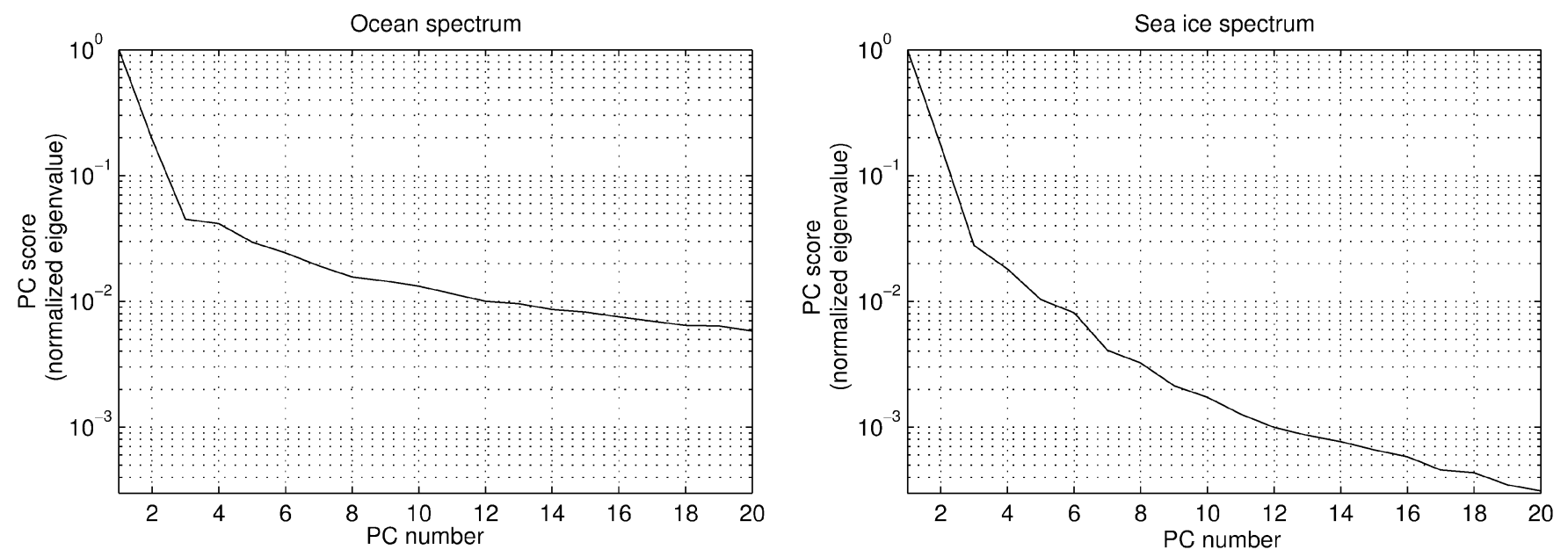

Fig. 2. First 20 PC scores of (left) Arctic ocean histograms and (right) Arctic sea ice histograms, illustrating the energy compaction in the top few PCs.

\section{B. Histogram Parameterization}

The discrimination parameters $\left[\mathrm{PR} A_{\mathrm{h}} V_{\mathrm{v}} V_{\mathrm{h}}\right]$ exhibit nearly Gaussian distributions throughout much of the year. However, anomalous weather conditions and fluctuating sea ice properties during the melt season cause sea ice and ocean statistics to deviate from Gaussian. The dynamic nature of the distributions of the discrimination parameters over sea ice-which are sometimes Gaussian, sometimes bimodal, and sometimes suggestive of Chi-squared distributions-present a challenge in multivariate statistical modeling. General statistical models which can account for higher order cumulants, such as the generalized lambda family of distributions [11], can be computationally intensive, and mathematically awkward to implement, especially in the multivariate case for which only an approximation may be constructed from contingency tables [12], [13].

The need for versatility elicits the use of an empirical model. Empirical models are potentially adaptive, but have several disadvantages: they are sensitive to noise, have less analytical value than a theoretical model, and have large memory requirements for finely binned data (for an $n$-dimensional histogram of $b$ bins for each dimension, the storage requirement is $b^{n}$ memory units).

Equation (5) requires two individual 4-D histograms of the discrimination parameter imagery - one for sea ice and one for ocean. For each histogram, it is desirable to reduce the storage requirement and misclassification induced bin height error (noise). This is achieved by parameterizing each histogram by a basis set of expected histograms.

The basis sets for sea ice and ocean histograms are generated by treating the histograms as images, and extracting the principal components (PCs) from a time-series ensemble of sea ice or ocean histograms via singular value decomposition (SVD). The resulting set of PCs span the space of observed sea ice or ocean histograms. The PCs allow the approximation of any sea ice or ocean distribution from a similar time period using only a few parameters. The parameters are the scaling coefficients of each PC vector which define the optimal (in a least-squares sense) reconstruction of the histogram using a linear combination of PC vectors. These are retrieved by mapping the histogram onto the truncated basis set via an inner product.

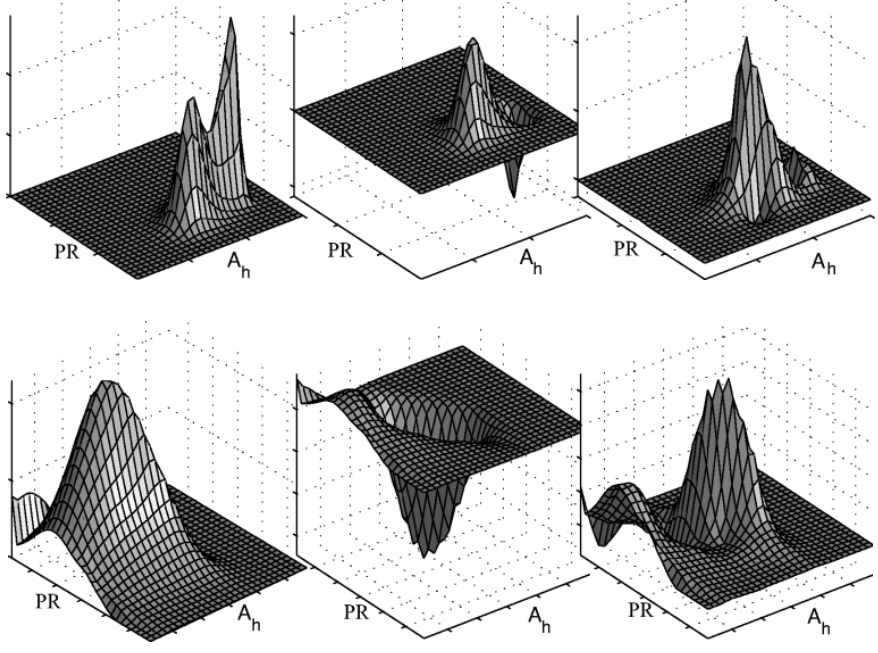

Fig. 3. First three PCs (ordered left to right) of Arctic ocean (top) and sea ice (bottom) histograms for the year 2001. Two-dimensional representations (PR versus $A_{\mathrm{h}}$ ) are shown.

Sea ice extent maps generated by the RL algorithm are used to train the algorithm. Four-dimensional histograms for sea ice and ocean are generated from this data for each day of the year 2001. The PCs of the time-series ensemble of histograms are found using the SVD, as mentioned above. The PC scores (the eigenvalues of the histogram ensemble's correlation matrix normalized by the largest eigenvalue) are shown for the Arctic sea ice and ocean in Fig. 2.

The first PC for sea ice and ocean represents the "average" component in the time-series ensembles of histograms. For this reason, the first PC in both cases dominates. It is also evident that the Arctic sea ice PC scores fall off more rapidly than the ocean PC scores. This is due to high variations in ocean histograms due to ocean storms.

Slices of the first three PCs for Arctic ocean and sea ice are shown in Fig. 3. The first PC for ocean histograms (the "average" histogram) is Gaussian shaped, and the first PC for Arctic sea ice histograms is bimodal.

In operation, histograms are generated for sea ice and ocean via sea ice extent maps that may contain misclassification errors. 

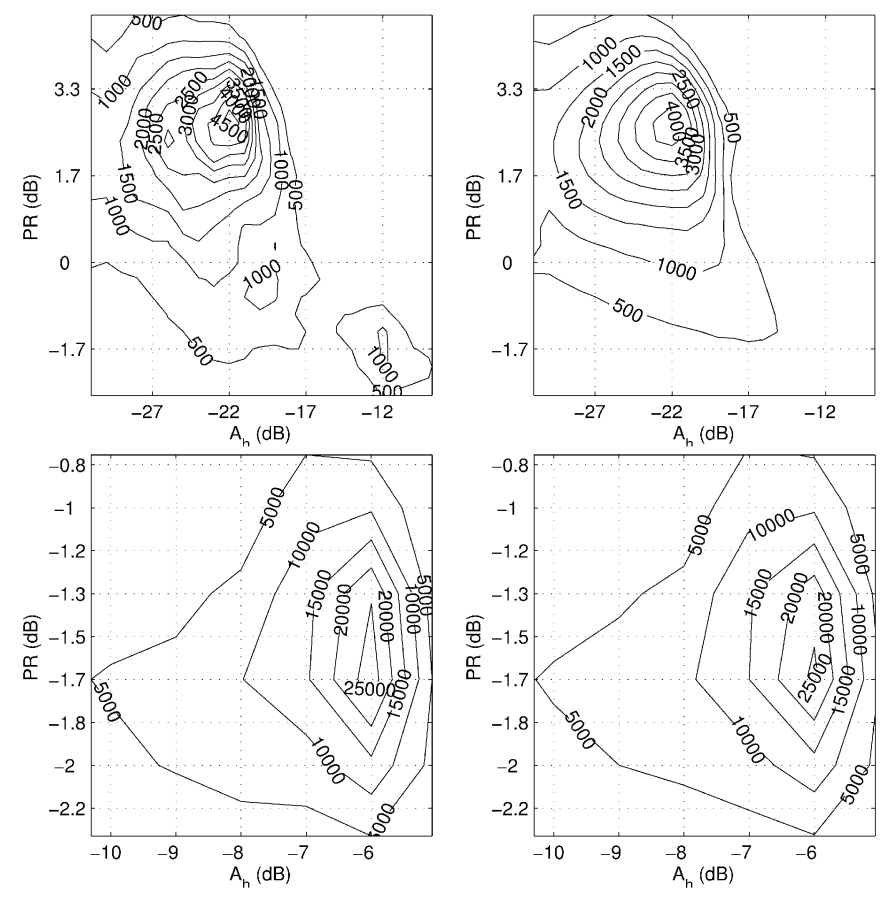

Fig. 4. Histogram parameterization using (top row) Arctic ocean histogram PCs and (bottom row) Arctic sea ice histograms. The original histogram is shown in the left column, and the parameterized histogram in the right column. The ocean histogram, which includes some sea ice contamination (contours in the lower right of the subfigure), is filtered in the parameterizing process. The sea ice histogram is not significantly modified as a result of parameterizing the sea ice histogram. Contour labels are pixel counts.

The resulting sea ice histograms contain ocean contamination, and the ocean histograms contain sea ice contamination. These "noisy" histograms are parameterized using a truncated set of the sea ice/ocean histogram PCs. The histogram reconstructed from PCs conforms to the ensemble of sea ice and ocean histograms used to generate the basis set, i.e., the truncated PC basis set functions as a filter to eliminate random errors in the "noisy" histogram's shape (we note, however, that systematic errors may not be filtered). An example of this noise-reduction property is shown in Fig. 4. Histograms which already conform to the set of expected shapes do not experience significant distortion through the reconstruction process (see Fig. 4). It is important to note that the parameterized histogram is only an estimate of the true histogram. The estimate may be improved by noting that bin heights for each bin must be greater than zero. Hence, negative bin heights are set equal to zero. Also, population size is generally not maintained in the parameterizing process. If the relative population sizes are known, the parameterized histograms may be normalized to reflect this knowledge. These modifications to the parameterized histograms have been implemented in Fig. 4.

In summary, histograms are selected to model dynamic sea ice and ocean statistics. Since histograms are sensitive to contamination caused by misclassifications, the histograms are parameterized by the PCs of sea ice and ocean histograms of previously classified images that are computed offline in an algorithm training step. The histogram parameterization filters out shape distortions caused by random misclassification in the histogram. Using this model for both the Arctic and the Antarctic, the dynamic nature of polar sea ice and ocean distributions are cap- tured in a way that rejects contamination, but maintains shape diversity.

\section{Iterative Bayes Detection Algorithm}

The Bayes detection approach is used iteratively to produce polar sea ice extent maps from SeaWinds data. First, an initial estimate of sea ice extent is produced using histograms for sea ice and ocean from the previous day. Loss maps $L_{01}^{x, y, t}$ and $L_{10}^{x, y, t}$ are generated from ice extent maps from the previous day. Here, it is assumed that the statistics and spatial distribution for sea ice and ocean do not change significantly in a single day, except for sea ice fluctuations around the ice edge (where we set $L_{01}^{x, y, t}$ equal to $L_{10}^{x, y, t}$ ).

The initial estimate may contain classification errors. To reduce the number of misclassified pixels, the initial classification is used to generate statistical and spatial information for another iteration of the classifier. This process is repeated until convergence is reached.

We note that the initial classification is on a individual pixel basis with each pixel independently classified. To improve the spatial homogeneity of the sea ice classification a simple method is implemented in the iteration process. It is based on the idea that if the observation $\mathbf{z}$ has been flagged as sea ice, then the neighbors of $\mathbf{z}$ are likely to also be sea ice. Hence, pixels in the neighborhood of $\mathbf{z}$ are considered candidates for classification as sea ice by including their values when computing the prefiltered sea ice histogram. This step is implemented prior to each iteration by dilating the classification map used to generate statistical information.

This spatial inclusion step aids in the method's ability to recover from poor statistical a priori information. In the case where initial sea ice distributions are too restrictive to include all of the true ice pixels, spatial inclusion helps to "broaden" the sea ice distribution in subsequent iterations. In the case where initial sea ice distributions are too broad, spatial inclusion potentially leads to even larger misclassified regions. Fortunately, spatial inclusion can be checked by proper selection of the loss maps $L_{01}^{x, y, t}$ and $L_{10}^{x, y, t}$.

Since initial loss maps $L_{01}^{x, y, t}$ and $L_{10}^{x, y, t}$ are constructed to allow for sea ice growth/retreat, the region of ignorance around the sea ice edge may be large. As noted previously, the loss maps do not influence the decision rule within this region. This can result in misclassifications if the discrimination parameters exhibit microwave signature anomalies within the region. To increase the efficacy of the loss maps as the algorithm nears convergence, the loss maps are updated at each iteration to reflect the new sea ice location from the most recent classification. The loss maps are also updated at each iteration so that the region of ignorance contracts (using progressively fewer dilations), accomplished through simulated annealing,

$$
\hat{L}_{i j}^{x, y, t} \leftarrow(1-\alpha) \hat{L}_{i j}^{x, y, t}+\alpha L_{i j}^{x, y, t}, \quad \alpha \in[0,1]
$$

where $\alpha$ is a forgetting factor, $\hat{L}_{i j}^{x, y, t}$ is the adaptive loss map, and $L_{i j}^{x, y, t}$ is a loss map created from the classification results of each iteration. To force the region of ignorance to contract, each new loss map $L_{i j}^{x, y, t}$ in the iteration sequence is created using fewer dilations of the sea ice classification map than the loss map of the previous iteration. The initial loss map is created 


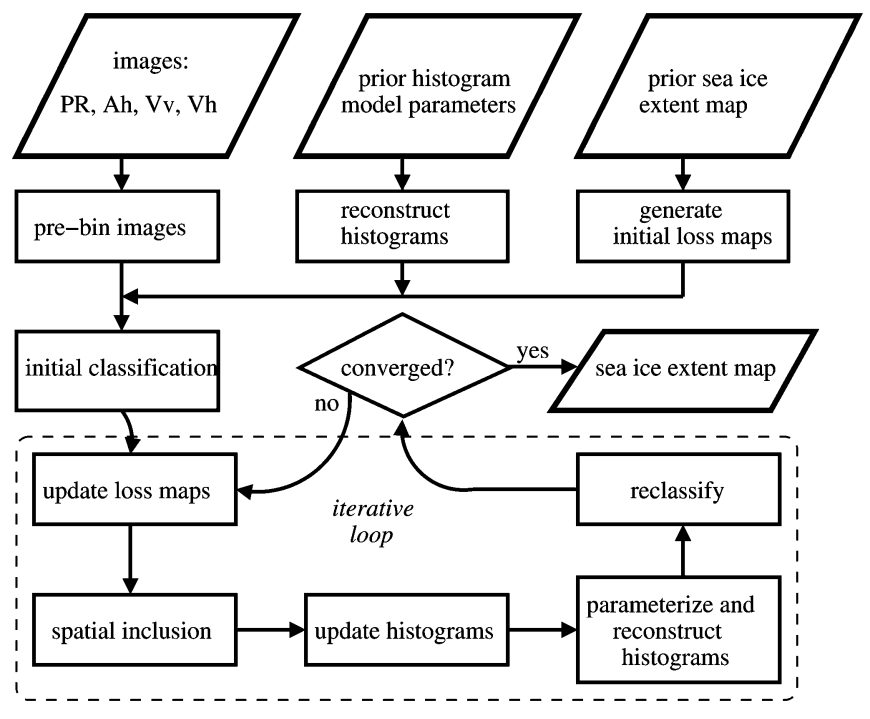

Fig. 5. Flowchart of the sea ice mapping algorithm when used with a time series of data. Discrimination parameter images are converted to histogram bin numbers in a prebinning step.

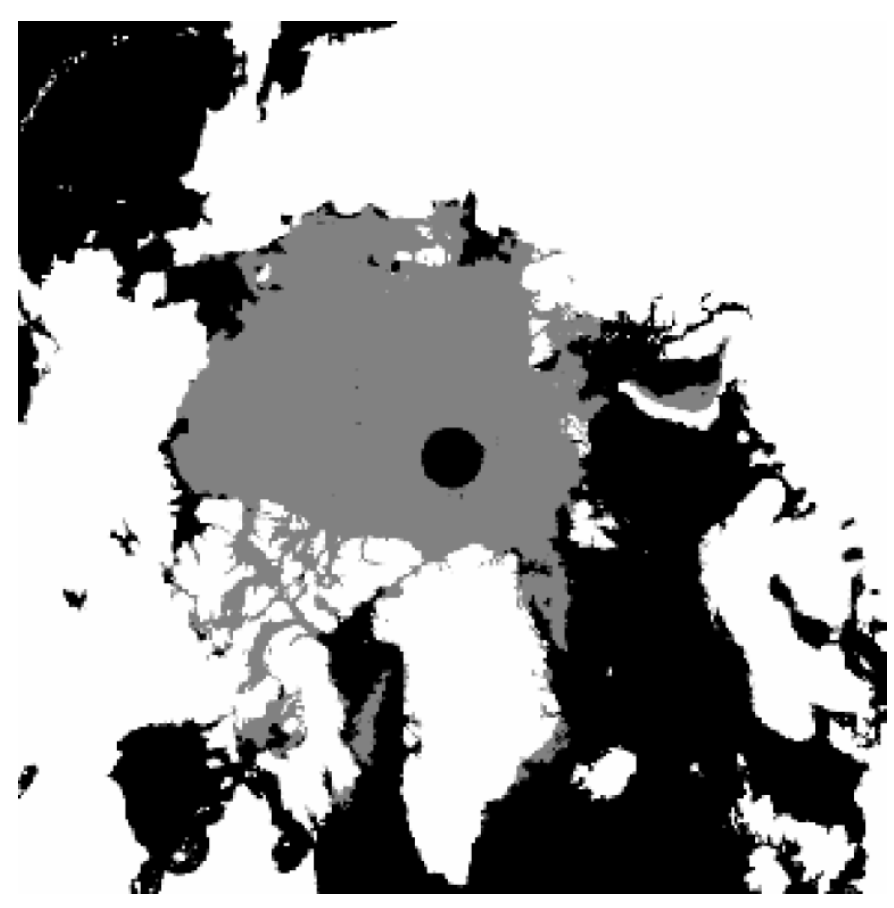

Fig. 6. Sample output of the sea ice mapping algorithm. Prior data were obtained via an ice extent map produced by the RL algorithm. Shown is the ice extent map for DOY 206, 2001.

from a dilated estimate of sea ice extent from a previous day, as described previously.

A diagram detailing the flow of the algorithm is shown in Fig. 5. A sample sea ice extent map for the Arctic region is shown in Fig. 6.

\section{Tuning Parameters}

The algorithm flowchart presented in Fig. 5 provides a highlevel view of the sea ice mapping process. Unlike the RL algorithm, the new method provides several tuning parameters which may be set to adjust the performance of the algorithm
TABLE I

Confusion Matrix of the Total SEA ICE AND Ocean PiXel Counts FOR THE ARCTIC REgION VERSUS THE RL ALGORITHM FOR 2001

\begin{tabular}{c||c|c||c}
\hline \multicolumn{1}{c||}{} & \multicolumn{2}{c||}{ New Algorithm } & \\
& sea ice & ocean & \% agreement \\
\hline \hline RL sea ice & 175466064 & 1228983 & $99.3 \%$ \\
\hline RL ocean & 4873032 & 202661025 & $97.7 \%$ \\
\hline
\end{tabular}

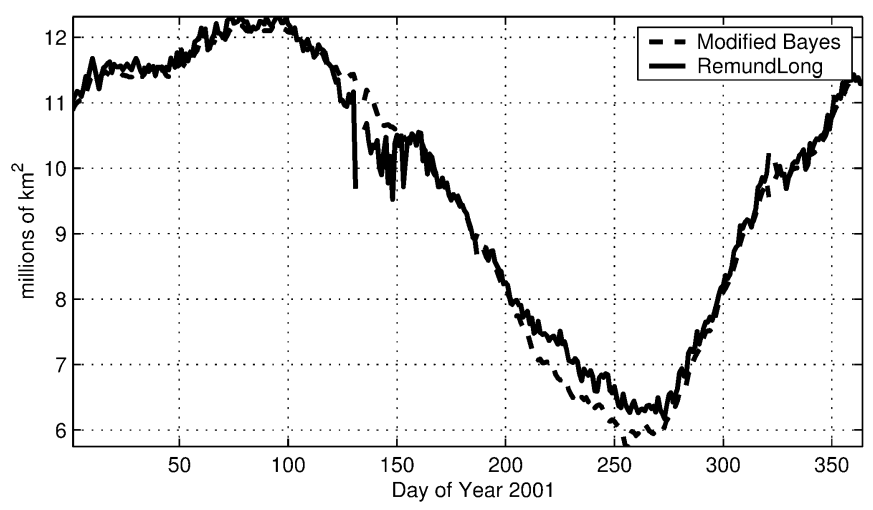

Fig. 7. Total Arctic sea ice area for 2001 reported by (black dashed) the modified Bayes algorithm and (gray solid) the RL algorithm for SeaWinds.

for a particular dataset. These tuning parameters are briefly discussed here.

1) Training Data (Histogram Principal Components): The algorithm may be trained to perform differently by proper computation of the PCs of sea ice and ocean histograms. This predisposes the algorithm to particular histogram shapes. In this study, the histograms used to calculate the PCs are generated using 50 RL sea ice maps from 2001 (spaced about seven days apart) to obtain a sample of the seasonal variations. These sea ice extent maps - which are subjectively validated - are used with PR, $A_{\mathrm{h}}, V_{\mathrm{v}}$, and $V_{\mathrm{h}}$ imagery to generate separate histograms for sea ice and ocean. From the resulting histograms, an orthogonal basis is extracted: the principal components. Since the ice masks needed in this training phase are used only to capture the general histogram shapes, performance is only weakly dependent on the training set.

2) Reconstruction Coefficients: The principal components used for the histogram basis set are ordered by the magnitude of the eigenvalue associated with the PC. While the full basis may be computed, the ordered basis set is truncated to a length that spans most of the space of the histograms. The accuracy of reconstructing histograms from principal component vectors may be adjusted by restricting the number of vectors used in reconstruction. This dictates to what extent histograms are smoothed by the PCs. For the Arctic region, 40 PCs enable accurate reconstruction of sea ice and ocean histograms; however, algorithm performance is relatively insensitive to the exact number of PCs, so long as a sufficient number is used to prevent oversmoothing.

3) Loss Map Dilation: The extent of dilation used when creating loss maps $L_{i j}^{x, y, t}$ limits how far we believe sea ice edge can move in a single day (for $n=0$ ), and to what extent we "trust" the classification map of the $n$th iteration (for $n>0$ ). Typically, loss maps are created initially using many dilations, and the region of ignorance is forced to contract by using successively fewer dialations, until the final iteration. For this study, 

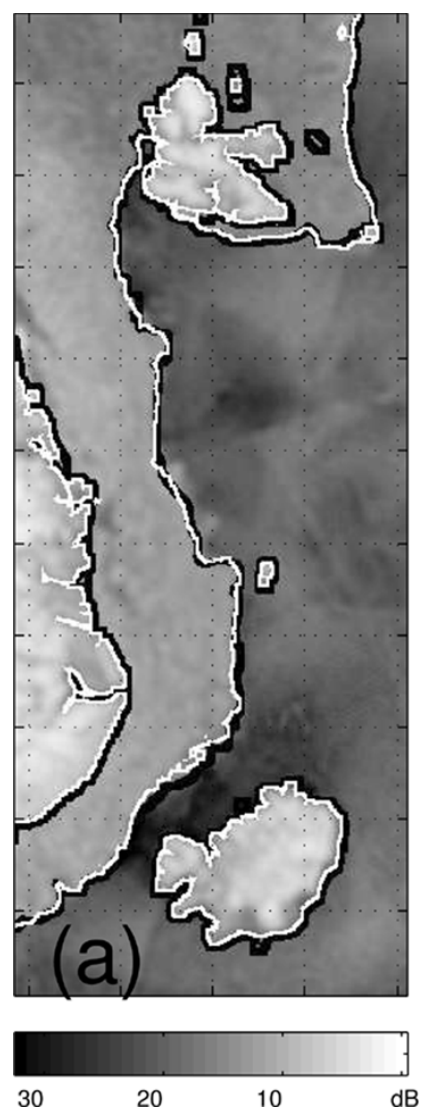
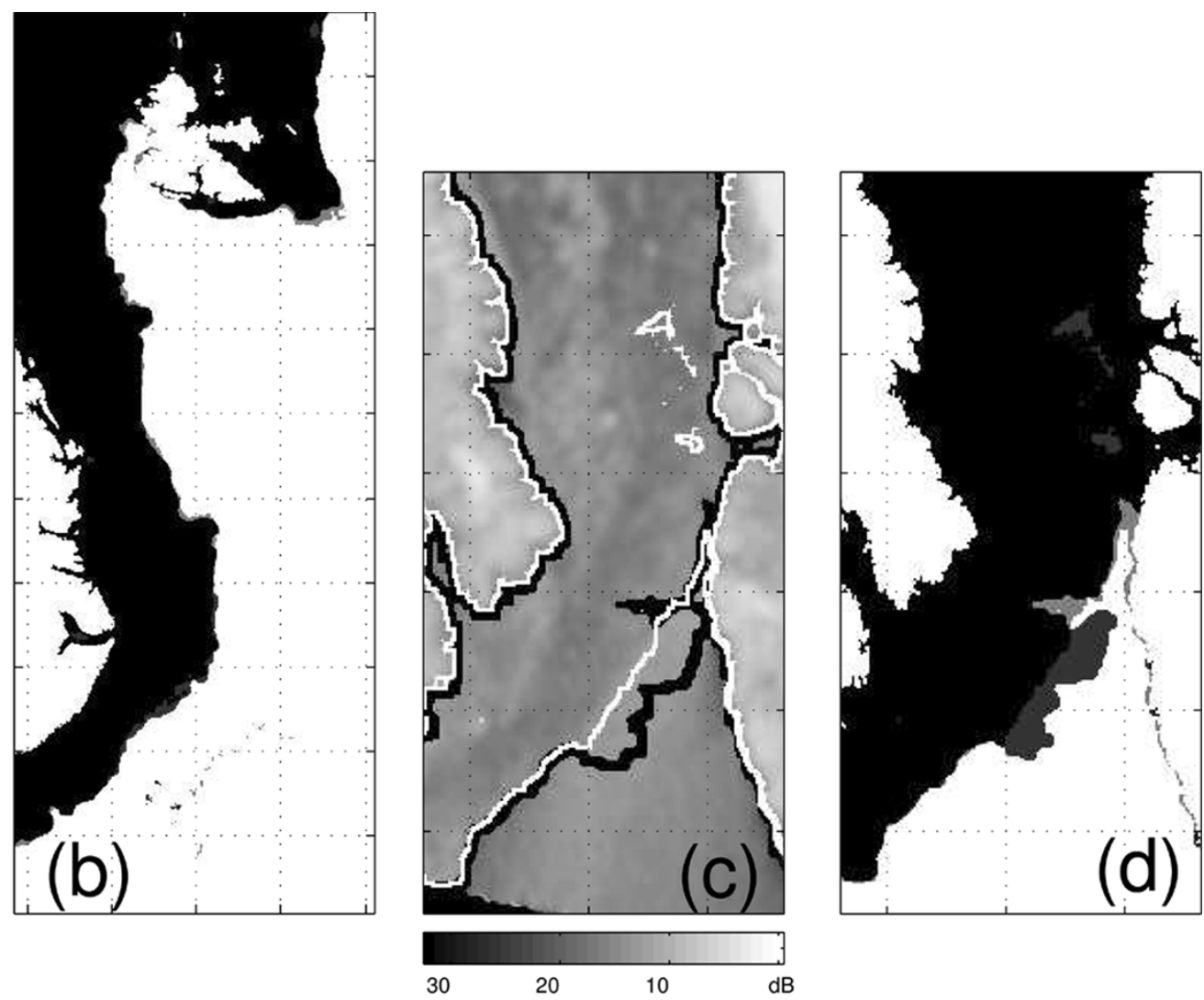

Fig. 8. (a) and (c) Ice edge comparison of (white line) modified Bayes and (black line) RL algorithms overlayed on $A_{\mathrm{h}}$ imagery. (b) and (c) Comparison of resulting masks where pixels that are common between the two algorithms are shown in black, pixels unique to the new modified Bayes algorithm are light gray, and pixels unique to the RL algorithm are dark gray. Data are for DOY 88, 2001. Center pixel for (a) and (b) is at $73.1^{\circ} \mathrm{N}, 8.75^{\circ} \mathrm{W}$ (east of Greenland), while the center pixel for (c) and (d) is at $66.6^{\circ} \mathrm{N}, 59.1^{\circ} \mathrm{W}$ (west of Greenland). Dotted grid lines are spaced $250 \mathrm{~km}$ apart.

the extent of dilation is decreased linearly from $40(178 \mathrm{~km})$ on the first iteration to $10(44.5 \mathrm{~km})$ at the last iteration.

4) Loss Map Erosion: If classification maps at the $n$th iteration contain misclassification errors (speckle noise), then the loss maps may contain corrupted loss values over a potentially large area, magnified by dilation operations. To ameliorate this, the loss maps are eroded prior to dilation (an unbalanced binary "opening" operation) by a factor based on the observed general maximum size of misclassified regions.

5) Loss Values: As discussed previously, the relative values of sea ice and ocean loss maps convey how much the classification map of the previous day (or previous iteration) is trusted. For convenience, loss values are restricted to $[0,1]$. In this study, high loss is given a value of unity and low loss a value of 0.05 .

6) Loss Map Forgetting Factor, $\alpha$ : The adaptive loss map forgetting factor $\alpha$ is also related to how much we trust classification maps of past iterations. For small values of $\alpha$, the algorithm is driven mostly by the current classification map. In this study, $\alpha$ is set to 0.2 .

7) Spatial Inclusion Dilation and Erosion: Spatial inclusion is implemented via an unbalanced binary opening operation (a small erosion followed by a larger dilation) applied to the classified image prior to updating the histograms. This promotes spatial homogeneity and helps the algorithm to recover from potentially poor prior data. The extent of dilation is related to how much we trust (or do not trust, rather) the loss map from the previous iteration. Large values compel the algorithm to incorporate a broader set of pixels when generating histograms.

The tuning parameter values used here have been selected somewhat arbitrarily to achieve reasonable performance over a seasonal cycle. Parameters could be further optimized to improve performance.

\section{RESULTS}

The technique described above is used to generate polar sea ice extent maps for the Arctic region for each day of 2001 from SIR egg data. As mentioned previously, training data are based on RL ice masks for 50 representative days spanning 2001. Since prior data are needed to "start" the algorithm, the RL ice map for the first day of 2001 is used for this purpose (i.e., the first estimate for day of year (DOY) 1, 2001 is the RL ice mask). Once started, in processing new data a three-sample temporal (noncausal) median filter is used on the new data sequence to reduce speckle noise while preserving spatial and temporal resolution. The length of this filter is short enough to insignificantly smooth temporal variability of a particular pixel—only in the rare cases does a pixel actually change states twice in a three-day window. The resulting ice extent maps are suitable for a variety of applications including wind retrieval, sea ice extent estimation, and sea ice motion studies. 
Validation of the sea ice extent estimates is difficult due to the lack of suitable comparison data. As an initial step toward a comprehensive validation in this section, the ice extent maps are compared to those produced by the RL algorithm, the Special Sensor Microwave/Imager (SSM/I) NASA Team algorithm, and RADARSAT-1 ScanSAR imagery.

\section{A. Comparison With the RL Algorithm}

Generally, the ice edge determined from the sea ice maps produced by the new method are visually highly correlated to the sea ice edge inferred from the RL algorithm for SeaWinds, as reported by the confusion matrix in Table I. Fig. 7 shows the total Arctic sea ice area reported by both algorithms as a function of the day of year. The overall discrepancy may be primarily ascribed to the fact that the new technique does not perform binary processing techniques to remove polynyas within the ice sheet, as does the RL algorithm (which overestimates the ice cover). The difference in the boreal summer (DOY 200-300) is due to the effect of surface melt events which are classified as ocean by the new algorithm, but as sea ice by the RL algorithm due to the binary processing phase. The discrepancy in the early spring (DOY 125-155) is due to a sensor outage and some misclassification by the RL algorithm caused by large-scale surface melting in the Hudson Bay.

A qualitative comparison of the performance of the two algorithms is difficult to assess. An empirical comparison shows that the algorithms perform quite similarly for much of the year. An illustrative example of the algorithms' performance is shown in Fig. 8. As shown, in cases where wind-agitated ocean waves near the sea ice edge cause ambiguity in the discrimination procedure for the RL algorithm, the modified Bayes algorithm is aided by spatial a priori information. In some instances, particularly during the summer, surface melting and sea ice motion cause high variance estimates in $V_{\mathrm{v}}$ and $V_{\mathrm{h}}$ imagery and cause both algorithms to perform poorly in areas of rapid change. As previously discussed, some of these surface melt events are classified as sea ice as a result of the binary processing phase of the RL algorithm.

The new algorithm presents several improvements over the RL algorithm:

1) There is a reduced dependence on post processing steps designed to eliminate spurious classification errors. The new method provides an unfiltered version of the SeaWinds ice edge, allows for the detection of polynyas, and allows the algorithm to track floes, icebergs, and large sections of sea ice which have been separated from the primary ice sheet.

2) The inclusion of spatial and statistical a priori information results in more consistent estimates of sea ice extent from day to day.

3) The adaptive statistical model allows for changes in sea ice and ocean properties without severely degrading the algorithm's performance.

These improvements come at a cost. First, unlike the RL algorithm, it is not possible to generate a sea ice extent map without a priori information. Thus, a different method must be used to "start" a sequence of sea ice extent estimates. Furthermore, the PCs must be calculated offline in a training phase before the algorithm can operate. Binary processing in the RL algorithm

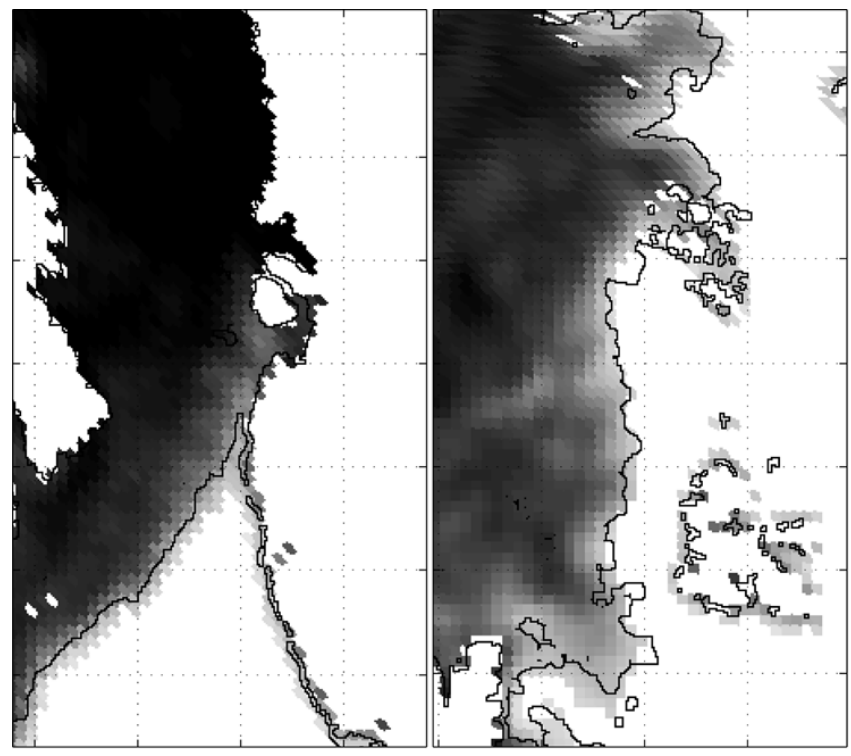

SSM/I NASA Team Sea Ice Concentration (\%)

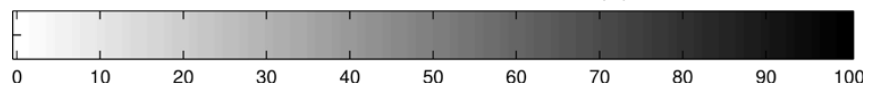

Fig. 9. Comparison of (black line) SeaWinds ice edge and SSM/I sea ice concentration for the Arctic (left) winter (DOY 88) and (right) summer (DOY 250). SSM/I concentration contours show consistency with the SeaWinds ice edge. The $0 \%$ to $100 \%$ sea ice concentration boundary may be fairly sharp in the winter case, but is blurred by ice/ocean spillover in the SSM/I footprint pattern. The left image is centered at $67.9^{\circ} \mathrm{N}, 55.8^{\circ} \mathrm{W}$; the right image at $82.2^{\circ} \mathrm{N}, 36.2^{\circ} \mathrm{E}$. Dotted grid lines are spaced $250 \mathrm{~km}$ apart.

corrects misclassification errors caused by the effects of surface melting on $V_{\mathrm{h}}$ and $V_{\mathrm{v}}$. This is not corrected, in general, by the new approach, unless the tuning parameters are set to minimize the effects of $V_{\mathrm{h}}$ and $V_{\mathrm{v}}$, which may come at the cost of losing information about polynyas. Third, the new method may be adversely affected by poor a priori information. This may be ameliorated, however, by adjusting the tuning parameters in the new method. The ability of the algorithm to recover from poor a priori information may be adjusted, but comes with the tradeoff of degraded performance, namely, more spurious misclassification errors, and less consistency between consecutive ice maps in a sequence. The ability to tune the algorithm may be viewed as both a strength and a weakness. Unlike the RL algorithm which is not tunable, but very robust, the new algorithm may be adjusted for desired performance, but may not be as robust for a particular set of tuning parameters.

\section{B. Validation: SSM/I NASA Team Sea Ice Concentration}

Results from the algorithm are compared to sea ice concentration estimates generated by the SSM/I NASA Team algorithm [14]. The latter are gridded on a polar stereographic projection at $25-\mathrm{km}$ pixel spacing. In order to compare datasets, the SSM/I concentration maps are interpolated onto the $4.45-\mathrm{km}$ spacing of the SIR polar stereographic projection. Fig. 9 shows the SeaWinds ice edge overlayed on SSM/I concentration data for both a winter and a summer case.

The average SSM/I sea ice concentration corresponding to the "edge" of the SeaWinds-derived ice extent map is extracted for each day of 2001 for the Arctic region. Ice edge concentration as a function of day is shown in Fig. 10. The comparison reveals seasonal dependence of the correlation between SSM/I 


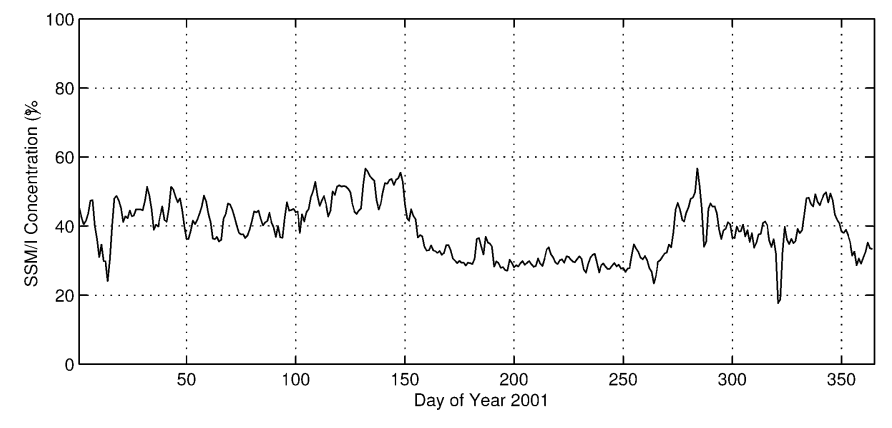

Fig. 10. Average Arctic SSM/I sea ice concentration corresponding to the ice edge derived from the new SeaWinds ice mapping approach.
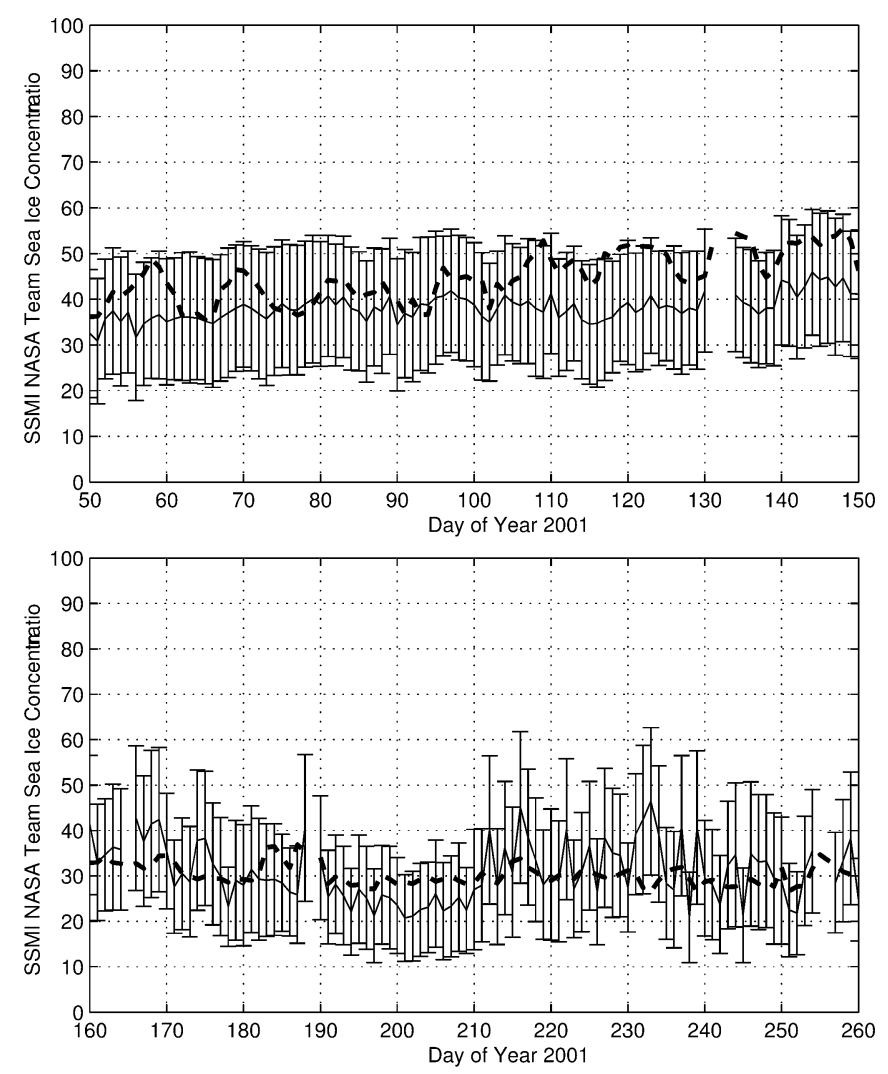

Fig. 11. Average Arctic SSM/I sea ice concentration corresponding to SeaWinds-derived ice edge for (top) DOY 50-150 (winter) and (bottom) DOY 160-260 (summer) 2001. Dashed line shows pretuning value, while solid line shows tuned value. Error bar heights are one standard deviation in ice concentration data.

sea ice concentration and the new SeaWinds ice edge, ranging from $30 \%$ in the summer to $50 \%$ in the winter. The peculiar sinusoidal behavior in the ice edge concentration during the first 150 days of 2001 is possibly due to sea ice growth, differences in sensor imaging times and intervals, and/or resolution and gridding differences in the imagery.

For clarity, separate segments of the resulting ice edge concentrations are shown in Fig. 11 for the winter and the melt season. Fig. 11 reveals that the winter SeaWinds sea ice edge corresponds to $40 \%$ to $50 \%$ sea ice concentration with large variability over the season. However, the variance of the SSM/I concentration at the ice edge for a given day is consistent throughout the season. This suggests that the SeaWinds-reported sea ice edge is consistent, and that the sinusoidal variations are either geophysical, or can be ascribed to the inaccuracy of SSM/I ice concentration readings at crisp ice/ocean edges [15]. In the winter, the ice edge is fairly abrupt, i.e., there may be a sudden change from a high concentration to zero concentration. The SSM/I instrument is sensitive to sea ice which may only partially fill the antenna footprint. This causes the transition between sea ice and ocean to appear more gradual than the true ice edge and the ice edge reported by the higher resolution SeaWinds imagery. During the summer, the sea ice edge is more diffuse and the SeaWinds-reported ice edge corresponds to the $30 \% \mathrm{SSM} / \mathrm{I}$ sea ice concentration contour.

To demonstrate the flexibility of the algorithm, the tuning parameters are adjusted in an effort to produce an ice map that corresponds to a lower SSM/I concentration contour. The number of PCs used in histogram smoothing is increased from 40 to 50, the loss map forgetting factor is increased from 0.2 to 0.6 , and the extent of dilation in the spatial inclusion step is increased from 3 to 4 . To extend the ice edge slightly, the ocean loss map is initially dilated ten pixels, while the ice loss map is eroded by five pixels. This creates a region of ignorance five pixels $(22.25 \mathrm{~km})$ wide that is offset by five pixels from the original ice edge. This influences the algorithm to create ice maps which extend further out. The choice of five pixels is somewhat arbitrary-it is based on expected ice growth, and is chosen to offset effects of sea ice motion on $V_{\mathrm{v}}$ and $V_{\mathrm{h}}$. This produces a region of influence five pixels wide around the original ice edge in which sea ice is more likely to be chosen by the classifier than ocean.

Fig. 11 shows the newly computed average SSM/I sea ice concentration for the winter period. The new ice edge concentration is less sporadic than the previously computed ice edge, and exhibits values which correspond more closely to $30 \%$ to $40 \%$ concentration rather than $40 \%$ to $50 \%$ concentration. The concentration for the summer shown in Fig. 11, however, is slightly more sporadic, but still correlates well with the $30 \%$ sea ice edge. This is a result of surface melting events, to which the radiometer is less sensitive relative to the scatterometer. The tuning parameters may be modified to some extent to match lower ice edge concentration values, but ultimately, the characteristics of the discrimination parameters govern how the algorithm performs.

\section{Validation: RADARSAT-1 ScanSAR}

Results from the algorithm are compared to RADARSAT-1 ScanSAR imagery for qualitative visual comparison. Comparison with the high-resolution data is difficult for two reasons. First, because of the low-coverage nature of SAR, usable RADARSAT-1 data at the ice edge is difficult to obtain. Thus, we have used only a handful of RADARSAT- 1 mosaics from various seasons to provide a representative comparison. Furthermore, the data are difficult to compare. RADARSAT-1 imagery is a "snapshot" of the ice edge at a particular time of day, while SeaWinds imagery is obtained over a 24-h period. As a result, especially during rapid ice growth or retreat, the ice edge inferred from the temporally averaged data may appear to be inconsistent with snapshot images. Instruments such as SeaWinds, which enjoys greater coverage, also suffer from blurring caused by sea ice motion during several satellite passes-hence, the reported sea ice edge should be thought of as the "average" sea ice edge during the imaging interval. Nonetheless, RADARSAT-1 data are an excellent source of 

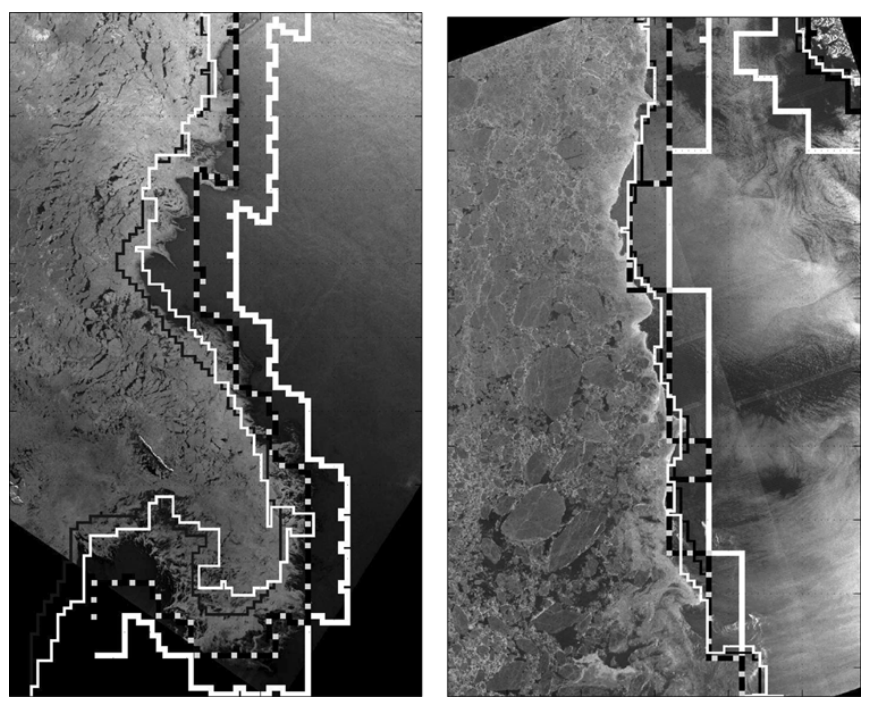

Fig. 12. (Left) RADARSAT-1 ScanSAR (uncalibrated) mosaic for DOY 12, 2001 east of Svalbard. The SSM/I 30\% contour (thick black/white dotted) is fairly consistent with the true edge, albeit at a coarse resolution. The SSM/I 10\% contour (thick white) is shown for comparison. The modified Bayes (thin white) and RL (thin gray) ice edges appear to be underestimates of the true ice extent. This may be partially due to sea ice growth and the difference in imaging times between SeaWinds and RADARSAT-1. The center pixel is at $76.9^{\circ} \mathrm{N}, 29.2^{\circ} \mathrm{E}$. (Right) A similar comparison for DOY 168, 2001 northeast of Greenland. The center pixel is at $79.2^{\circ} \mathrm{N}, 4.8^{\circ} \mathrm{W}$ (RADARSAT- 1 data (C) 2001, Canadian Space Agency).

high-detail in situ image data useful for ice edge validation. This comparison provides a qualitative check that the reported sea ice edge is consistent with the shape and features of the true ice edge [9].

Fig. 12 shows a RADARSAT-1 ScanSAR mosaic for DOY 12,2001 . During the winter, ice growth is expected and is especially evident at the bottom of the image where small fingers of sea ice are forming along the peninsula. Motion stress on the ice pack is apparent by the small cracks and fissures within the ice sheet. The ice edge derived from the new ice mapping approach is shown, as well as the RL-derived ice edge, the SSM/I NASA Team $30 \%$ contour, and the SSM/I NASA Team $10 \%$ contour. Here, the spill-over effects of the ice edge into the SSM/I antenna footprint over ocean is evidenced by the displaced $10 \%$ contour. The new SeaWinds ice edge and the RL-derived ice edge appear to underestimate the true ice edge-perhaps an effect of sea ice growth. The SSM/I 30\% provides a fairly consistent estimate of the RADARSAT-1 ice edge, albeit at a coarse resolution.

Fig. 13 shows a similar RADARSAT-1 mosaic with overlayed sea ice edge estimates. The mosaic was imaged during the melt season, as evidenced by the separated floes within the ice pack and the diffuse edge at the bottom of the image. However, the ice edge appears fairly abrupt at the top of the image. The new SeaWinds edge and the RL-derived edge show excellent correlation with the RADARSAT-1 ice edge. The $30 \%$ $\mathrm{SSM} / \mathrm{I}$ contour also shows good correlation within the resolution limitations.

Fig. 13 shows a RADARSAT- 1 mosaic of a large polynya located off the northwest coast of Greenland. At the bottomright of the image, the sea ice edge is somewhat diffuse. The $\mathrm{SSM} / \mathrm{I}$ contours appear to be valid representations of the diffuse

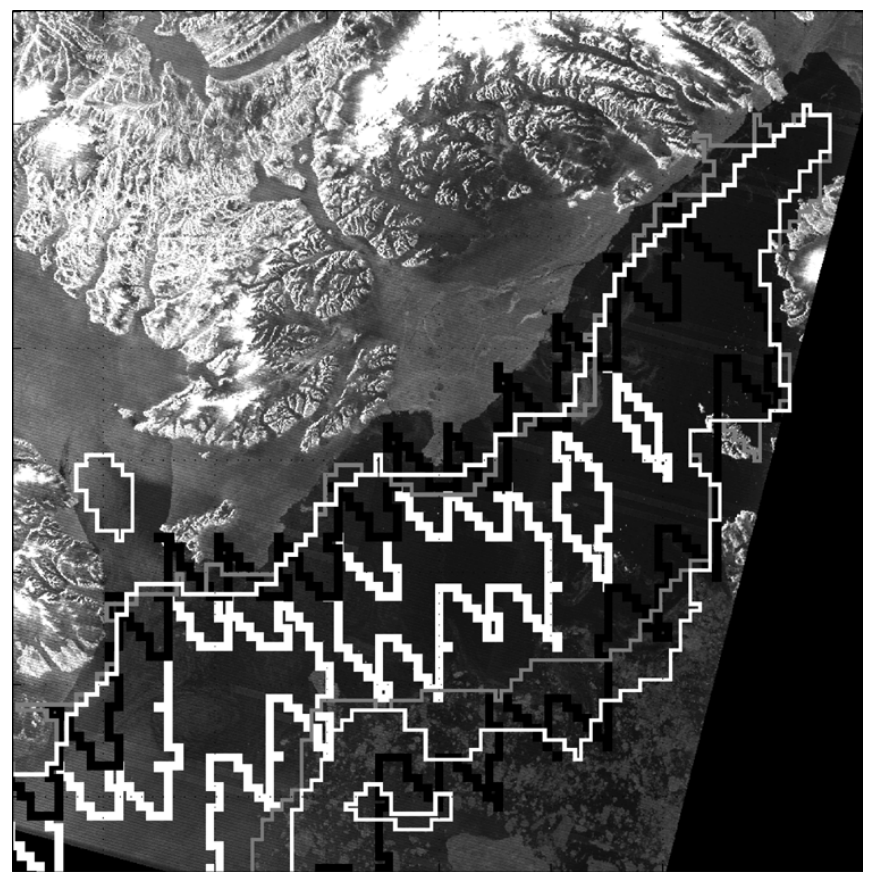

Fig. 13. RADARSAT-1 ScanSAR (uncalibrated) mosaic for DOY 169, 2001 showing the North Water Polynya just off the northwest coast of Greenland. The SSM/I 30\% contour (thick black) provides coarse detail of the polynya; the RL algorithm (thin gray) higher detail; the new method (thin white) identifies smaller polynyas (not geolocated) to the northwest and southeast. Each provides different variations of the diffuse ice edge at the bottom right of the image. The $\mathrm{SSM} / \mathrm{I} 10 \%$ contour (thick white) is shown for comparison. The center pixel is at $77.1^{\circ} \mathrm{N}, 73.2^{\circ} \mathrm{W}$ (RADARSAT-1 data (C 2001, Canadian Space Agency).

edge. The RL-derived ice edge shows excellent tracking of the diffuse edge, while the new SeaWinds edge follows the SSM/I $30 \%$ contour in the diffuse area. Both SeaWinds edges are good representations of the crisp RADARSAT-1 ice edge at the top of the image. The new method detects small polynyas that are disjoint from the large polynya.

Generally, sea ice maps derived from the new method correlate well with RL sea ice maps, the SSM/I NASA Team 30\% contour, and subjectively to RADARSAT-1 data (for SSM/I sea ice concentration data, the $30 \%$ contour shows the best correlation with the observed RADARSAT- 1 ice edges in the previous examples). The new SeaWinds edge shows detail on the scale of the resolution of the SeaWinds egg imagery, including small polynyas within the ice sheet.

\section{CONCLUSiON}

The RL algorithm for SeaWinds is another manifestation of the utility of Ku-band scatterometer data in polar sea ice detection. In an effort to reduce the dependence on binary processing routines, improve statistical modeling, and incorporate spatial and statistical a priori information, this new sea ice mapping algorithm for SeaWinds has been developed.

The new method requires a training phase in which typical sea ice and ocean histogram shapes are learned from previously computed sea ice masks. In addition, the method requires a priori estimates of the sea ice extent and ice/ocean histograms, e.g., computed by the RL algoroithm. However, once the algorithm has been trained, and a priori data are provided for the first day in a time-series, the algorithm can then run independently. 
The new technique shows good correlation with the RL algorithm ice edge, but exhibits several improvements. First, the algorithm produces ice maps at the effective resolution of the SIR imagery, but has few misclassification errors. This reduces the need for a binary processing phase that is a required part of the RL algorithm. The independence from binary processing routines allows the algorithm to track sea ice bodies that are disjoint from the primary ice sheet. Second, the inclusion of spatial and statistical a priori information provides for more consistent sea ice extent maps from day to day. Also, the more sophisticated statistical modeling approach facilitates the dynamic nature of polar sea ice and ocean microwave signatures.

While only limited data are available for validation, the resulting sea ice maps show high correlation with estimates from other sensors. In particular, the new SeaWinds ice edge corresponds to the $30 \% \mathrm{SSM} / \mathrm{I}$ NASA Team sea ice concentration contour. RADARSAT-1 ScanSAR imagery verifies the accuracy of the algorithm.

Although the algorithm presented in this work is designed for the SeaWinds scatterometer, it may be adapted for other instruments. This can be accomplished by changing the discrimination parameters and/or a priori information. Or, the discrimination parameters can be changed to enable for multisensor classification such as joint radiometer and scatterometer sea ice maps, benefiting from the advantages of both types of microwave sensors. The binary classification approach may also be extended to M-ary classification, such as ice type classification. These topics are left for future research.

\section{ACKNOWLEDGMENT}

QuikSCAT data were obtained from the Physical Oceanography Distributed Data Archive (PO.DAAC) at the Jet Propulsion Laboratory, Pasadena, CA. We thank the anonymous reviewers for their helpful comments and suggestions.

\section{REFERENCES}

[1] F. D. Carsey, Ed., Microwave Remote Sensing of Sea Ice. Washington, DC: Amer. Geophys. Union, 1992.

[2] Q. P. Remund, D. G. Long, and M. R. Drinkwater, "An iterative approach to multisensor sea ice classification," IEEE Trans. Geosci. Remote Sens., vol. 38, pp. 1843-1856, Mar. 2000.

[3] Q. P. Remund and D. G. Long, "Sea ice mapping algorithm for QuikSCAT and SeaWinds," in Proc. IGARSS, vol. 3, Jul. 1998, pp. $1686-1688$.

[4] — "Sea-ice extent mapping using Ku-band scatterometer data," $J$. Geophys. Res., vol. 104, no. C4, pp. 11 515-11 527, 1999.

[5] M. R. Drinkwater and D. G. Long, "Seasat, ERS-1/2 and NSCAT scatterometer observed changes on the large ice sheets," in Proc. IGARSS, vol. 4, 1998, pp. 2252-2254.

[6] JPL, QuickSCAT Science Data Product User's Manual, T. Lungu, Ed. Pasadena, CA: Jet Propulsion Lab., 2001.
[7] D. S. Early and D. G. Long, "Image reconstruction and enhanced resolution imaging from irregular samples," IEEE Trans. Geosci. Remote Sens., vol. 39, no. 2, pp. 291-302, Feb. 2001.

[8] D. G. Long, P. Hardin, and P. Whiting, "Resolution enhancement of spaceborne scatterometer data," IEEE Trans. Geosci. Remote Sens., vol. 31, no. 3, pp. 700-715, May 1993.

[9] J. Haarpaintner, R. T. Tonboe, D. G. Long, and M. L. VanWoert, "Automatic detection and validity of the sea ice edge: An application of enhanced resolution QuikSCAT/SeaWinds data," IEEE Trans. Geosc. Remote Sens., vol. 42, no. 7, pp. 1433-1443, Jul. 2004.

[10] T. K. Moon and W. C. Stirling, Mathematical Methods and Algorithms for Signal Processing. Upper Saddle River, NJ: Prentice-Hall, 2000.

[11] Z. A. Karian and E. J. Dudewicz, Fitting Statistical Distributions: The Generalized Lambda Distribution and Generalized Bootstrap Methods, 1st ed. Boca Raton, FL: CRC, 2000.

[12] R. L. Plackett, "A class of bivariate distributions," Biometrika, vol. 9, pp. 159-315, 1965.

[13] K. V. Mardia, "Some contributions to contingency-type distributions," Biometrika, vol. 54, pp. 235-249, 1967.

[14] D. Cavalier. (1996) NASA Team Sea Ice Algorithm. [Online]. Available: http://nsidc.org/data/docs/daac/nasateam/index.html.

[15] T. Agnew and S. Howell, "The use of operational ice charts for evaluating passive microwave ice concentration data," Atmos.-Ocean 41, vol. 4, pp. 317-331, 2003.

Hyrum S. Anderson (S'03) received the M.S. degree in electrical engineering from Brigham Young University (BYU), Provo, UT, in 2003.

$\mathrm{He}$ is currently researching at the Lincoln Laboratory, Massachusetts Institute of Technology, Lexington.

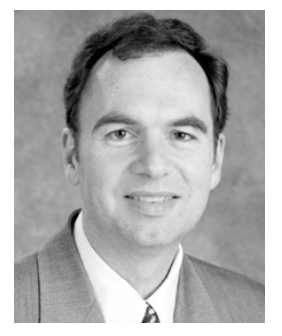

David G. Long (S'80-SM'98) received the Ph.D. degree in electrical engineering from the University of Southern California, Los Angeles, in 1989.

From 1983 to 1990 , he was with the National Aeronautics and Space Administration (NASA) Jet Propulsion Laboratory (JPL), Pasadena, CA, where he developed advanced radar remote sensing systems. While at JPL, he was the Senior Project Engineer on the NASA Scatterometer (NSCAT) project, which was flown aboard the Japanese Advanced Earth Observing System (ADEOS) from 1996 to 1997. He was also the Experiment Manager and Project Engineer for the SCANSCAT scatterometer (now known as SeaWinds). In 1990, he joined the Department of Electrical and Computer Engineering, Brigham Young University (BYU), Provo, UT, where he currently teaches upper division and graduate courses in communications, microwave remote sensing, radar, and signal processing, is the Director of BYU's Center for Remote Sensing, and is the Head of the Microwave Earth Remote Sensing Laboratory. He is the Principal Investigator on several NASA-sponsored interdisciplinary research projects in microwave remote sensing and innovative radar systems. He has numerous publications in signal processing and radar scatterometry. His research interests include microwave remote sensing, radar, polar ice, signal processing, estimation theory, and mesoscale atmospheric dynamics. He has over 250 publications in the open literature.

Dr. Long has received the NASA Certificate of Recognition several times. $\mathrm{He}$ is an Associate Editor for the IEEE GEOSCIENCE AND REMOTE SENSING LETTERS. 